\title{
Evaluating inherently safer design alternatives using hazard conflict approach
}

\author{
R. Rusli \& A. M. Shariff \\ Process Safety Research Group, Chemical Engineering Department, \\ Universiti Teknologi PETRONAS, Perak, Malaysia
}

\begin{abstract}
The application of Inherent Safety (IS) principles and Inherently Safer Design (ISD) concept has been proven to reduce the risk of accidents and is economically attractive for chemical process plants. However, they also suffer from several trade-offs or conflicts that arise from the modification suggested by the concept. A design which is identified to be inherently safer from one hazard could possibly alter the magnitude of other hazards, which were previously not at a critical level. Therefore, an IS tool should emphasise this limitation before a decision can be made, in order to obtain the best ISD alternatives. This paper presents a likelihood tool to evaluate the potential hazard conflicts in ISD alternatives at preliminary design stage. The tool is part of a framework which is developed based on risk approach. The proposed tool is applied to hydrogen storage systems with the objective to prevent and minimise potential fire and explosion. The results have shown that the new tool is able to highlight the potential new hazards and variation of magnitude of existing hazards which emerged when the hydrogen storage is changed according to the ISD concept at preliminary design stage.
\end{abstract}

Keywords: hazard conflicts, inherent safety, inherently safer design, risk analysis, preliminary process design.

\section{Introduction}

Risk reduction measures to eliminate or minimise consequences in chemical process industries can be achieved through four main strategies i.e. inherent, passive, active and procedural strategies [1]. The recommended first step is inherent strategies which can be defined as strategies that design out the hazard 
from the process or system using ISD concept. The ISD concept aims to eliminate the sources of harm by using less hazardous chemicals, smaller inventories of chemicals and milder process conditions. Thus, the changes in a design can exclude the inherent hazard and its potentially severe consequences instead of controlling it through add-on safety systems and procedures. However, in practice, a change that reduces one hazard may create a new one or increase the magnitude of another existing one [2]. This poses a challenge for designers as this trade off does not give the designers any room for errors in implementing the inherent strategies. Development of a safety tool that is capable of addressing the above issue is essential during the preliminary design stage when screening of design options is required.

Most of the early works in developing IS tools used a hazard-based approach within the studied process to evaluate inherent safety characteristics of different process options such as Prototype Index of Inherent Safety (PIIS) [3] and Inherent Safety Index (ISI) [4]. Then, the development of the tools focused on consequence-based approach such as the Integrated Inherent Safety Index (I2SI) by Khan and Amyotte [5]. Another consequence-based tool has been proposed by Tugnoli et al. [6] to assess the inherent safety of process alternatives using the concept of key performance indicators. Other tools such as the integrated risk estimation tool (iRET) by Shariff et al. [7], Inherent Safety Index Module (ISIM) by Leong and Shariff [8] and Process Route Index (PRI) by Leong and Shariff [9] were developed to evaluate process design alternatives on potential impact from consequences of vapour cloud explosion through the integration of process design simulator with enhanced ISI. The integration efforts in these tools are developed to ease design modifications at an early stage of the design. Furthermore, Shariff and Leong [10] proposed a preliminary inherent risk assessment (IRA) technique to evaluate the amount of risk which is inherent to the properties of the chemicals and process condition. Recently, Shariff and Zaini [11] have extended the iRET capability to evaluate toxic releases at early design stage.

All of the above tools can roughly indicate which option is relatively an inherently safe. However, a hazard-based method may not be the ultimate decision making tool to select the best ISD options as the likelihood of hazard conflicts due to design modification is not fully evaluated in this approach. To overcome this limitation, a risk based approach is proposed in this paper to evaluate inherently safer process design alternatives. However, the present paper will discuss the hazard conflict approach only due to space constraint.

\section{Likelihood index of Hazard conflict (LIHC)}

The main objective of LIHC is to identify the possibility of hazard conflicts which could cause failure or uncontrollable hazard and resulted in the increase of the overall risk of accident in the final stage of design. LIHC is the integrated index unit in quantifying risk in order to alert process designers on the safety level of the process after the inherent strategies are considered in the design. Figure 1 shows the overall framework to estimate the risk index, namely 


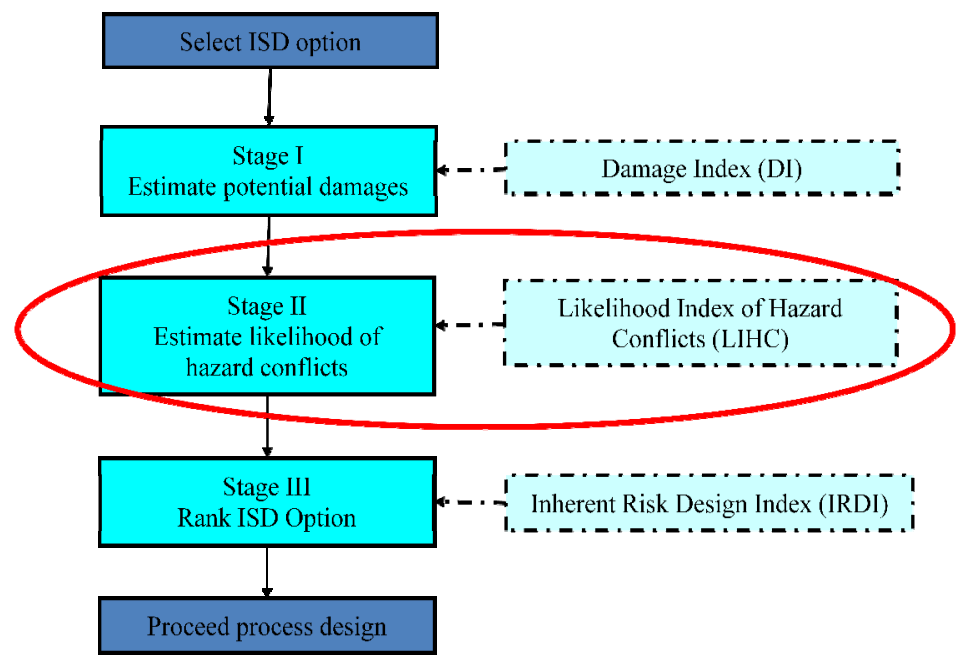

Figure 1: $\quad$ LIHC as part of QIISD framework.

Quantitative Index of ISD (QIISD). A brief discussion will be given on QIISD while the procedure to estimate LIHC will be the main focus of the present paper.

QIISD is developed using a risk-based approach through the identification of consequence and likelihood to obtain Inherent Risk Design Index (IRDI). Damage Index (DI) represents the potential consequence from the hazardous energy contained in the process. LIHC is determined after quantifying the order of hazard magnitude due to changes in the targeted process safety criteria through the estimation of Likelihood Index of Design is Inherent Safer (LIDIS). The estimation of IRDI and LIHC option-i (op-i) are based on Rusli [12] as given in eqns. (1) and (2):

$$
\begin{aligned}
\operatorname{IRDI}_{\mathrm{op-i}} & =\mathrm{DI}_{\mathrm{op}-\mathrm{i}} \bullet \mathrm{LIHC}_{\mathrm{op}-\mathrm{i}} \\
\text { LIHC }_{\mathrm{op}-\mathrm{i}} & =\left(1-\text { LIDIS }_{\mathrm{op-i}-\mathrm{i}}\right)
\end{aligned}
$$

\subsection{Likelihood index of design is inherent safer (LIDIS)}

LIDIS is a likelihood index that accounts the degree of safeness in an ISD option with regard to Inherent Safety principles proposed by Kletz [13] such as substitute, minimise, moderate and simplify. In addition, these principles are used to trigger the potential hazard conflicts exist between the principles. For example, one ISD option proposed a smaller type of continuous reactor (application of minimise principle) instead of a batch reactor but may require high temperature and pressure (conflict in application of moderate principle). Furthermore, this option may require frequent transportation due to constraints in the inventory of the materials (conflict in application of simplify principle). For each Inherent Safety principle, a group of relevant process safety criteria is set to 
indicate the principle as shown in Table 1. This arrangement is made to further stimulate any potential conflicts between hazards and contradiction on the complexities of safety for the overall plant. For example, one ISD option proposed to use a less toxic solvent (application of substitute principle for toxicity criteria) but may have a lower boiling point that could lead to the possibility of a pressure hazard due to a boiling solvent in the event of a runaway reaction (conflict in application of substitute for flammability criteria). Since the scope of this study is at the preliminary design stage, the process safety criteria are limited to chemical and physical properties of the substances, initial process conditions and preliminary design data of the process units. These inputs are typically available in process flow diagram (PFD) and preliminary equipment design.

Table 1: Inherent safety principles and process safety criteria to estimate LIDIS [12].

\begin{tabular}{|c|c|c|c|}
\hline $\begin{array}{l}\text { Inherent } \\
\text { Safety } \\
\text { Principles }\end{array}$ & $\begin{array}{c}\text { Target } \\
\text { Characteristics }\end{array}$ & \multicolumn{2}{|r|}{ Process Safety Criteria } \\
\hline Substitute & $\begin{array}{l}\text { Quality of } \\
\text { materials used } \\
\text { or produced }\end{array}$ & \multicolumn{2}{|c|}{$\begin{array}{l}\text { Hazardous of substances = NFPA ranking on flammability, } \\
\text { explosive, reactivity and toxicity for feed, product and by- } \\
\text { product }\end{array}$} \\
\hline Minimise & $\begin{array}{l}\text { Quantity of } \\
\text { process } \\
\text { inventory }\end{array}$ & \multicolumn{2}{|c|}{$\begin{array}{l}\text { Volume = percent accumulated in vessel and intermediate } \\
\text { storage, amount of gas release, concentration }\end{array}$} \\
\hline \multirow{2}{*}{ Moderate } & \multirow{2}{*}{$\begin{array}{l}\text { Operating and } \\
\text { safe limit } \\
\text { conditions }\end{array}$} & $\begin{array}{l}\text { Thermal } \\
\text { Runaway }\end{array}$ & $\begin{array}{l}\text { Temperature effect = adiabatic } \\
\text { temperature rise, time to maximum rate of } \\
\text { runaway } \\
\text { Pressure effect = vapour pressure, amount } \\
\text { of solvent evaporated }\end{array}$ \\
\hline & & $\begin{array}{l}\text { Fire and } \\
\text { Explosion }\end{array}$ & $\begin{array}{l}\text { Temperature effect = flash point, } \\
\text { flammability limits, } \\
\text { Pressure effect = fraction liquid vaporised, } \\
\text { pressure build-up }\end{array}$ \\
\hline \multirow{3}{*}{ Simplify } & \multirow{3}{*}{$\begin{array}{l}\text { Easiness in the } \\
\text { design and } \\
\text { operating }\end{array}$} & $\begin{array}{l}\text { Controllability: } \\
\text { basic } \\
\text { requirement }\end{array}$ & $\begin{array}{l}\text { Basic controls in flow, temperature, } \\
\text { pressure, level etc. }\end{array}$ \\
\hline & & $\begin{array}{l}\text { Controllability: } \\
\text { technical } \\
\text { requirement }\end{array}$ & $\begin{array}{l}\text { Advance technical control measures such } \\
\text { as emergency cooling, quenching and } \\
\text { flooding, depressurisation etc. }\end{array}$ \\
\hline & & $\begin{array}{l}\text { Complexity on } \\
\text { overall process } \\
\text { unit and plant }\end{array}$ & $\begin{array}{l}\text { Number of vessels, auxiliary units, } \\
\text { frequency of transportation, complexity in } \\
\text { maintenance etc. }\end{array}$ \\
\hline
\end{tabular}


The computation of LIDIS for one option, LIDIS $_{\mathrm{op}}$, are developed in this research which is expressed by the yield between the actual Likelihood Score $\left(\mathrm{LS}_{\mathrm{act}}\right)$ and the maximum $\mathrm{LS}_{\max }$ that the option should achieve as shown in eqns. (3) and (4), respectively [12]:

$$
\begin{aligned}
& \operatorname{LIDIS}_{\text {op }}=\frac{\mathrm{LS}_{\text {act }}}{\mathrm{LS}_{\text {max }}} \\
& \mathrm{LS}_{\text {max }}=N \bullet(+10)
\end{aligned}
$$

where $N$ is the total number of process safety criteria used from all IS principles in a particular option.

For an option, the actual score, $\mathrm{LS}_{\text {act, }}$, is derived from the summation of Total Likelihood Score (TLS) of all IS principles. The TLS for each principle is estimated by adding the Process Factor Score (PFS) of each process safety criteria in the individual IS principle as illustrated in eqns. (5) and (6), respectively [12]:

$$
\begin{gathered}
\mathrm{LS}_{\mathrm{act}}=\mathrm{TLS}_{\mathrm{sub}}+\mathrm{TLS}_{\text {min }}+\mathrm{TLS}_{\mathrm{mod}}+\mathrm{TLS}_{\text {sim }} \\
\mathrm{TLS}_{\mathrm{j}}=\sum_{\mathrm{i}=\mathrm{m}}^{\mathrm{n}} \mathrm{PFS}_{\mathrm{i}}
\end{gathered}
$$

where the subscripts $j, i, n$, sub, min, mod and sim refer to principle $j$, process factor score $\mathrm{i}$, process safety criteria $\mathrm{m}$ and $\mathrm{n}$, substitute, minimise, moderate and simplify, respectively.

A guideline to indicate the order of the hazard conflict is developed using an index approach with increment of 1 from +10 to -10 . If the LIDIS is positive conflict, it shows that the ISD option has reduced the hazard contributed by the process safety criteria. Subsequently, when the LIDIS is at negative value, it shows that the ISD option has increased the hazard of the process safety criteria which indirectly reveals the potential of hazard conflicts introduced by the ISD option. In addition, the reduction or increment of the hazard conflict is revealed not only within the main process unit but also the related site-process units such as auxiliary units, storages and transportations. Therefore, the difference in each process safety criteria between a base case and one ISD option is estimated using eqns. (7) and (8), respectively [12]:

$$
\begin{aligned}
& \mathrm{PFS}_{\mathrm{i}}=\operatorname{Max}\left[-10,\left(1-\frac{\mathrm{psc}_{\mathrm{op}}}{\mathrm{psc}_{\mathrm{bc}}}\right) \times(10)\right] \quad \text { if } \mathrm{psc}_{\mathrm{op}}>\mathrm{psc}_{\mathrm{bc}} \\
& \mathrm{PFS}_{\mathrm{i}}=\operatorname{Min}\left[10,\left(1-\frac{\mathrm{psc}_{\mathrm{bc}}}{\mathrm{psc}_{\mathrm{op}}}\right) \times(-10)\right] \text { if } \mathrm{psc}_{\mathrm{op}}<\mathrm{psc}_{\mathrm{bc}}
\end{aligned}
$$

where the subscript i refers to Process Factor Score (PFS) i; $\mathrm{psc}_{\mathrm{op}}$ is the process safety criteria for the ISD option and $\mathrm{psc}_{\mathrm{bc}}$ is the process safety criteria for the base case. 
The Likelihood Score of the listed process safety criteria for substitute, minimise and moderate as in Table 1 is estimated using the actual value obtained from the design option. However, the estimation of Likelihood Score for simplify principle $\left(\mathrm{LS}_{\mathrm{sim}}\right)$ which represents the complexity in process safety controls requirement, layout, handling and transportation need to refer to the developed guidelines as shown in Table 2 and 3. These guidelines are required since some of the process safety criteria are relatively subjective and limited information available at early stage of design. Fundamental basic design calculations, literatures and also expert judgements could be used to assist the determination of the likelihood score.

Table 2: Guidelines of $\mathrm{LS}_{\text {sim }}$ for basic and advance controls requirement [12].

\begin{tabular}{|c|c|}
\hline Description & Index value \\
\hline Essential & 10 \\
\hline Very important & 9 \\
\hline Important & 8 \\
\hline Not important but required & 7 \\
\hline Required & 6 \\
\hline Requirement is moderate & 5 \\
\hline Good if available & 4 \\
\hline Requirement does not affect process & 3 \\
\hline Not required & $1-2$ \\
\hline
\end{tabular}

Table 3: Guidelines of $\mathrm{LS}_{\text {sim }}$ for complexity and handling of process unit [12].

\begin{tabular}{|c|c|c|}
\hline Process Complexity & Description & Index value \\
\hline \multirow{2}{*}{$\begin{array}{c}\text { Agitator } \\
\text { Auxiliary unit; compressors, } \\
\text { pumps }\end{array}$} & Essential & 10 \\
\cline { 2 - 3 } Multi-unit, parallel, length of \\
\cline { 2 - 3 } piping, & Very important & 9 \\
\cline { 2 - 3 } $\begin{array}{c}\text { Storages } \\
\text { Frequency of handling } \\
\text { Mode of transportation }\end{array}$ & Important & 8 \\
\cline { 2 - 3 } & Not important but required & 6 \\
\cline { 2 - 3 } & Required & 5 \\
\cline { 2 - 3 } & Requirement is moderate & 3 \\
\cline { 2 - 3 } & Good if available & $1-2$ \\
\hline
\end{tabular}

Table 4: $\quad$ Likelihood ranking for LIHC.

\begin{tabular}{|c|c|c|}
\hline \multicolumn{2}{|c|}{$\begin{array}{c}\text { Likelihood Index of Hazard } \\
\text { Conflicts (LIHC) }\end{array}$} & Possibility of hazard conflicts \\
\hline Low (L) & $0-0.59$ & $\begin{array}{c}\text { Design is inherently safer } \\
\text { Possible hazard conflicts are fewer and manageable } \\
\text { through standard process safety procedures }\end{array}$ \\
\hline Medium (M) & $0.60-1.09$ & $\begin{array}{c}\text { Design has similar degree of hazards with the base case } \\
\text { Possible of hazard conflicts are critical and redesign } \\
\text { may be required }\end{array}$ \\
\hline High (H) & $1.10-2.0$ & $\begin{array}{c}\text { Design is not inherently safer } \\
\text { Possible hard conflicts are highly critical and } \\
\text { redesign is highly recommended }\end{array}$ \\
\hline
\end{tabular}


The end result of LIHC is described through 3 levels; Low (L), Medium (M) and High $(\mathrm{H})$ levels to illustrate the possibility of hazard conflicts in the ISD option in comparable to the base case design (Table 5). These levels are developed in this paper to show that the possibility of hazard conflicts would lead to the uncontrollable stage of the hazard either by creating new hazards or escalating the current existing ones. Therefore, the ISD option is considered as ideally inherently safer when it has attained the lowest LIHC at 0 because the possibility of hazard conflicts in this option is highly unlikely. Then, the ISD option that has LIHC less than 1 shows that ISD option would probably have fewer hazard conflicts. The ISD option with LIHC equivalent to 1 is expected to have similar degree of potential hazards with the base case design which could have been transferred to the other parts of the plant. Finally, the ISD option which is regard as not inherently safe is the ISD option that obtained the LIHC of more than 1 to the highest LIHC at 2. This LIHC value demonstrates that the modification proposed by the ISD option would create substantial hazard conflicts by critically increases the hazards in the process unit or other systems in the plant.

Table 5: $\quad$ Summary of features and process conditions for $\mathrm{H}_{2}$ storage systems [14].

\begin{tabular}{|c|c|c|c|c|}
\hline $\begin{array}{c}\text { Features and process } \\
\text { conditions }\end{array}$ & Compressed & Cryogenic & $\begin{array}{c}\text { Metal } \\
\text { hydride }\end{array}$ & $\begin{array}{c}\text { Complex } \\
\text { hydride }\end{array}$ \\
\hline Technology & Commercial & Commercial & Research & Research \\
\hline Pressure $(\mathrm{MPa})$ & 25 & 0.6 & 1.1 & 0.1 \\
\hline Temperature $(\mathrm{K})$ & 300 & $20 / 25$ & 300 & 300 \\
\hline $\mathrm{H}_{2}$ mass stored per unit $(\mathrm{kg})$ & 35.7 & 500 & 105 & 500 \\
\hline Number of units & $\begin{array}{c}2 \text { tube trailers } \mathrm{x} \\
7 \text { units }\end{array}$ & 1 & 5 & 1 \\
\hline
\end{tabular}

Table 6: The LIHC for hydrogen storage systems with compressed system as the base case.

\begin{tabular}{|c|c|c|c|}
\hline ISD Option & LIHM & Level & Design Criticality Descriptions \\
\hline Cryogenic & 1.07 & Medium & $\begin{array}{c}\text { Design has similar degree of hazards with the base case } \\
\text { Possible of hazard conflicts are critical and redesign may } \\
\text { be required }\end{array}$ \\
\hline $\begin{array}{c}\text { Metal } \\
\text { hydrides }\end{array}$ & 1.34 & High & $\begin{array}{c}\text { Design is not inherently safer } \\
\text { Possible hazard conflicts are highly critical and redesign } \\
\text { is highly recommended }\end{array}$ \\
\hline $\begin{array}{c}\text { Complex } \\
\text { hydrides }\end{array}$ & 1.44 & High & $\begin{array}{c}\text { Design is not inherently safer } \\
\text { Possible hazard conflicts are highly critical and redesign } \\
\text { is highly recommended }\end{array}$ \\
\hline
\end{tabular}

\section{Application of LIHC to hydrogen storage system}

To illustrate the tool presented here, a hydrogen safety study reported by Landucci et al. [14] for hydrogen storage systems at medium-scale is used as the 
case study with the objectives to identify the inherently safer storage system. There are four type of hydrogen storage techniques considered in this case study: (i) storage of hydrogen gas under pressure; (ii) storage of liquefied hydrogen; (iii) storage as a metal hydride; and (iv) storage as a complex hydride. The same assumptions and conditions used by Landucci et al. [14] for medium-scale storage are applied here in order to demonstrate the applicability of the developed tool. Table 6 shows the summary of features and process conditions for all type of hydrogen storage systems. The simplified process flow diagram for all hydrogen storage systems and brief descriptions of each process systems can be obtained from Landucci et al. [14]. To facilitate the evaluation, the process flow diagram of the storage technologies are reviewed comprehensively with the support of other literatures related to the hydrogen process and safety issues [15-20].

\subsection{Results and discussions}

The literatures show that the first two techniques of hydrogen storage systems are considered as the conventional technologies that are globally used such as refineries and chemical plants. On the other hand, the last two techniques are still under research and development and have been indicated as possible inherently safer alternatives $[21,22]$. Based on the above information, a potential loss of containment due to hydrogen released would be high in compressed and cryogenic storage system while metal hydrides and complex hydrides are considered as the inherently safer alternatives. The best principles to represent these design changes are through the application of substitute and moderate principles. The hazardous material, such as hydrogen, is substituted with hydrides material and the hazardous operating conditions in the compressed and cryogenic system are moderated with less hazardous conditions. This implies that metal and complex hydrides technologies are inherently safer for the storage of hydrogen, as stable hydride in solid phase is comparable to compressed and cryogenic storage system.

The estimation of LIDIS is carried out by setting the compressed system as the base case because this system has hazardous operating conditions and higher inventory of hydrogen. This can be confirmed via quantification of the consequence for this process. However, detailed estimation of the potential consequence is not the focus of this paper. The selection of process safety criteria is based on the potential to contribute to the possibility of accidental human fatality and structural damages, such as by system failure, ruptured storage tank, leakage, etc. The LIDIS results for all ISD options are reported in Figure 2 with the compressed system as the base case. The evaluations made for each of the IS principles, are explained here.

The potential conflicts identified for the substitute principle are the potential hazards of the metal and complex hydrides themselves. The NFPA ranking for these materials are classified as flammable, reactive, and toxic, with levels of hazard at 3,2, and 2, respectively. In addition, all of these materials were judged to be flammable, pyrophoric, and water reactive, which requires extra safety precautions when handling them [20]. This has resulted in high conflicts to the 
already highly hazardous characteristics of hydrogen, which are released by the system at $1.1 \mathrm{MPa}$ and supplied to the compression unit. For the cryogenic storage system, since the only hazardous material is hydrogen, thus, the hazardous characteristics in this system are at the same level as the compressed system.

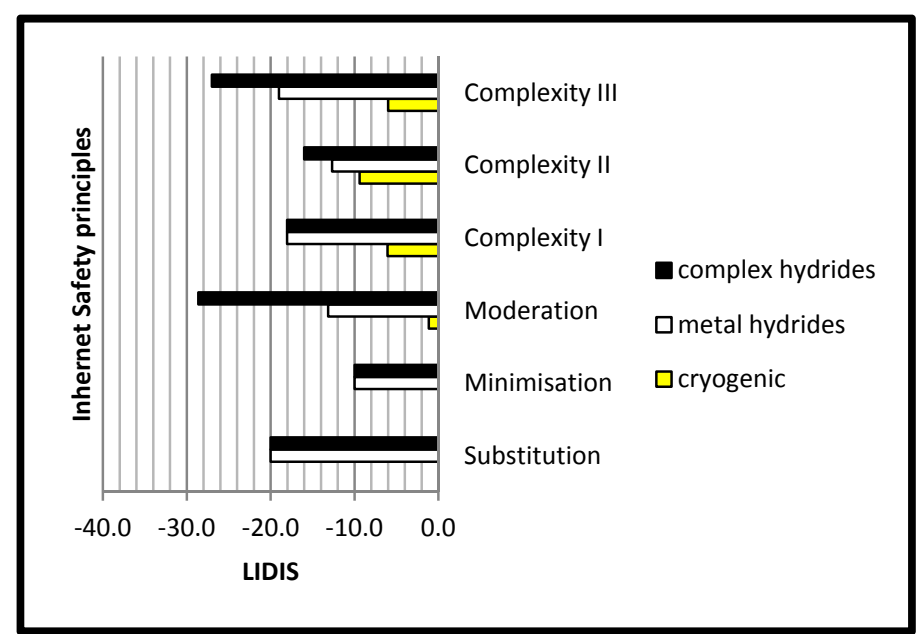

Figure 2: $\quad$ LIDIS results for all options based on IS principles.

For the minimise principle, the inventory of hydrogen in all types of storage system has been maintained at the same amount of $500 \mathrm{~kg}$. Although the metal hydrides storage systems involved only $100 \mathrm{~kg}$ of hydrogen to be discharged per adsorption unit, the multi-units of adsorption ( 5 units) by metal hydrides are considered to be an equivalent amount of hydrogen to be discharged from the process. In fact, the amount of metal hydrides in the adsorption unit has affected this principle as being the potential negative conflict, since minimise principle does not only apply to hydrogen, but also to other potentially hazardous materials involved in the system.

When the same principle applies to complex hydrides, the same conflicts as metal hydrides are identified; which refer to the bulk inventory of the complex hydrides in slurry that needs to be supplied to the semi-batch reactor. As a result, the inventory is increased for a complex hydrides storage system, due to a requirement to have additional bulk storage units, before the discharge of hydrogen via the reactor. Therefore, the risk associated with a catastrophic rupture and leak at the feed pipeline, is high in both of the storage systems, as reported by Landucci et al. [14].

The potential conflicts in the moderate principle, focuses on the severity of the operating conditions. The positive conflict of metal and complex hydrides is illustrated as moderate operating conditions in the system, where the instability of hydrogen storage can be minimal. However, the LIHC tool has revealed that there is a potential for fire and dust explosion in both metal and complex 
hydrides, due to their pyrophoric properties and other safety parameters, such as burning rate, minimum ignition energy, and maximum rate of pressure rise, as testified by Zalosh [19] and Tanaka et al. [20]. These properties could inherently affect the process when there is a potential of a worst case scenario, such as system failure, human error, etc. in the handling of the storage system. Besides that, the compression unit in both systems also contribute to the negative conflicts under the moderate principle, which causes the storage systems to be at a par with compressed and cryogenic systems.

Finally, the result of the potential hazard conflicts based on the simplify principle, shows the highest total index values for both metal and complex hydrides in comparison with compressed and cryogenic systems. These negative conflicts come mainly from the complexity of hydride technologies itself, which may have problems to maintain the reliability of the auxiliary units, such as the heat exchanger, compressor, and piping connections between the secondary equipment, in order to complete the process. Moreover, the complexity of these storage systems may require additional safety control measures, such as secondary containment, since the system could contain heavy hydrides slurry, as in complex hydrides. Besides that, the bulk storage unit, semi-batch reactor, and compression unit, may require blast walls to protect from the potential of a dust explosion. For transportation activities, as described above, metal and complex hydrides are flammable, pyrophoric, and water-reactive materials, which define the incompatibility of these materials, resulting in them being classified as United Nations Packing Group I; as the most stringent category of container regulations for transporting these materials [20]. This finding is equal to the evaluation which contributes to moderate negative conflicts, but still the transportation of compressed and cryogenic hydrogen is far more hazardous than the other two options.

After consideration of LIDIS, the LIHC results showed that complex hydrides storage has the highest potential conflicts as illustrated in Table 7. It can be concluded that the complex hydrides storage system appeared to have low consequence of loss containment, since the operating conditions of this system are at a moderate level compared to others. However, this storage system contained the highest potential of hazard conflicts such as the possibility of dust explosions, the complexity of the design, and the transporting and handling process. Therefore, the tool demonstrates that the design is at HIGH level, and the designer should look into the identified conflicts and carry out detailed study in order to further enhance the overall inherent safety if this storage system is selected as the best ISD alternative.

\section{Conclusion}

The proposed tool is able to indicate the potential risk based on the hazard conflicts associated with ISD design options. The risk of hazards is believed to be apparent through a combination of IS principles as the guidewords in the developed tool. Thus, it helps the end-user in the decision making process to identify the best ISD that will give better impact in risk reduction of hazards and 
low in potential of new hazards. In addition, the tool is able to highlight possible risk transfer not only within the design of the process unit but the potential risk at site-process units, which is highly relevant to support the main process unit. The developed tool could give greater impact and better sensitivity to process changes and later transform the results to risk values for better comparison at early design stage.

\section{Acknowledgements}

The authors would like to acknowledge the management of Universiti Teknologi PETRONAS for the support given in terms of funds and research materials throughout the research works. The authors also gratefully acknowledge Prof Faisal I Khan from Memorial University, Canada for his valuable input and support in this work.

\section{References}

[1] Bollinger, R.E., Clark, D.G., Dowell, I.R.M., Ewbank, R.M., Hendershot, D.C., Lutz, W.K., Meszaros, S.I., Park, D.E. \& Wixom, E.D., Inherently Safer Chemical Processes: A Life Cycle Approach, Wiley-AIChE, New York, 1997.

[2] CCPS, Guidelines for Risk Based Process Safety, Center for Chemical Process Safety/AIChE, New York, 2007.

[3] Edwards, D.W., Lawrence, D. \& Rushton, A.G., Quantifying the inherent safety of chemical process routes, Fifth World Congress of Chemical Engineering. 2, pp. 1113-1118, 1996.

[4] Heikkilä, A.M., Inherent safety in process plant design, $\mathrm{PhD}$ Thesis of University of Helsinki, Finland, 1999.

[5] Khan, F.I. \& Amyotte, P.R., Integrated inherent safety index (I2SI): A tool for inherent safety evaluation, Process Safety Progress, 23, pp. 136-148, 2004.

[6] Tugnoli, A., Cozzani, V. \& Landucci, G., A consequence based approach to the quantitative assessment of inherent safety, AIChE Journal, 53, pp. 3171-3182, 2007.

[7] Mohd Shariff, A., Rusli, R., Leong, C.T., Radhakrishnan, V.R. \& Buang, A., Inherent safety tool for explosion consequences study, Journal of Loss Prevention in the Process Industries, 19, pp. 409-418, 2006.

[8] Leong, C.T. \& Shariff, A.M., Inherent safety index module (ISIM) to assess inherent safety level during preliminary design stage, Process Safety and Environmental Protection. 86, 113-119, 2008.

[9] Leong, C.T. \& Shariff, A.M., Process route index (PRI) to assess level of explosiveness for inherent safety quantification, Journal of Loss Prevention in the Process Industries, 22, pp. 216-221, 2009.

[10] Shariff, A.M. \& Leong, C.T., Inherent risk assessment-A new concept to evaluate risk in preliminary design stage, Process Safety and Environmental Protection, 87, pp. 371-376, 2009. 
[11] Shariff, A.M. \& Zaini, D., Toxic release consequence analysis tool (TORCAT) for inherently safer design plant, Journal of Hazardous Material, 182, pp. 394-402, 2010.

[12] Rusli, R. Integrated inherently safer design evaluation tool (IISDET) for use in preliminary design stage, $P h D$ Thesis, Universiti Teknologi PETRONAS, 2011.

[13] Kletz, T.A. Inherently safer plants, Plant/Operating Programme, vol.4, pp.164-167. 1985.

[14] Landucci G., Tugnoli A. and Cozzani V., 2008, Inherent safety key performance indicators for hydrogen storage systems. Journal of Hazardous Materials, 159. pp. 554-566, 2008.

[15] Conte M., Prosini P.P \& Passerini S., Overview of energy/hydrogen storage: state-of-the-art of the technologies and prospects for nanomaterials, Material Science Engineering B, 108, pp. 2-8, 2004.

[16] Zhou L., Progress and problems in hydrogen storage methods, Renewable \& Sustainable Energy Reviews, 9, pp. 395-408, 2005.

[17] Sarkar A. and Banerjee R., Net energy analysis of hydrogen storage options, International Journal of Hydrogen Energy, 30, pp. 867-877, 2005.

[18] Kinzey B.R., Ruiz A. \& Davis P.B., The Hydrogen safety program of the U.S. Department of Energy, Proc. of the 1st International Conference on Hydrogen Safety, Pisa. 2005.

[19] Zalosh R., Metal hydride fires and fire suppression agents. Journal of Loss Prevention in the Process Industries, 21. pp.214-221, 2008.

[20] Tanaka H., Tokoyoda K., Matsumoto M., Suzuki Y., Kiyobayashi T. \& Kuriyama N., Hazard assessment of complex hydrides as hydrogen storage materials. International Journal of Hydrogen Energy, 34, pp. 3210-3218, 2009.

[21] Browning, D., Jones, P. \& Packer, K., An investigation of hydrogen storage methods for fuel cell operation with man-portable equipment, J. Power Sources, 65, pp. 187-195. 1997.

[22] Aiello, R., Matthews, M.A., Reger, D.L. \& Collins, J.E., Production of hydrogen gas form novel chemical hydrides, International Journal of Hydrogen Energy, 24, pp. 1123-1130, 1999. 